\title{
Research on Molding Technologies of Bamboo/Plastic Composite
}

\author{
Kun Li $i^{a}$, Lianfa Yang ${ }^{b^{*}}$ \\ School of Mechanical \& Electrical Engineering, Guilin University of Electronic Technology, \\ Guilin, Guangxi, 541004, China \\ aLiKunAndrew@163.com, by-lianfa@163.com
}

Keywords: Bamboo/plastic composite, Molding technology, Future focuses.

Abstract. Bamboo/plastic composite (BPC) is an advanced composite material. BPC has been used in building, gardening, outdoor products and other fields due to its advantages of biodegradable, high mechanical properties, corrosion resistance, low cost and so on. In this paper, four BPC molding methods are presented. The process of molding and technical characteristics are explained, and how did the molding affects the properties of BPC are introduced. Also this paper proposes the future focuses of BPC molding.

\section{Introduction}

BPC is an advanced composite material which holds advantages of low cost, high strength, biodegradable. And it has been used in building, gardening, outdoor products and other fields [1]. But the performance of BPC is largely influenced by molding, and the performance has indirect effects the promote use of BPC. So, it is particularly important to researches on molding technology of bamboo plastic composite. In this paper, four BPC molding methods are presented. The process of molding and technical characteristics are explained, and how did the molding affects the properties of BPC are introduced. Also this paper proposes the future focuses of BPC molding.

\section{Compression Molding}

Compression molding (Also known as hot-press molding) is a molding method by which material is shaped and solidified in the pressing of mold under molding temperature. As illustrated in Fig. 1, the mold is open and cleared, after BPC is placed into the die cavity. Then, after the punch and the die are closed, the material is shaped in the mold under the pressure and the temperature for a period of time. Finally, the mold is opened again and the element is taken out from the mold by the ejector rod, and the flash is removed which produced by the material clearance.

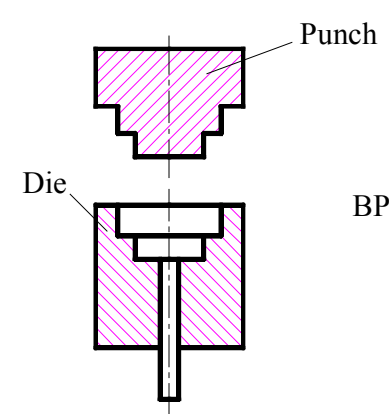

(a)

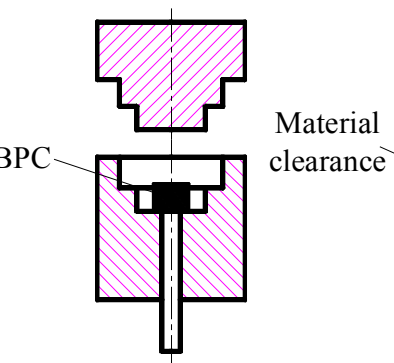

(b)

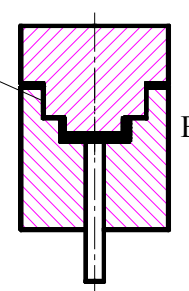

(c)

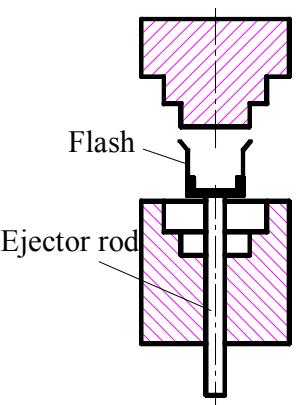

(d)

Fig.1 Schematic drawing of compression molding: (a) opening and clearing mould,

(b) placing BPC, (c) molding and (d) removing workices.

$\mathrm{H}$. Ismail et al [2] studied the impact of loadings of bamboo fiber on the mechanical properties of BPC by using hot-press molding to press the experimental samples. H Wang et al [3] investigated 
the effect of the proportion between bamboo and Polyvinyl Chloride (PVC), bamboo particle size, and moisture content on the stability and mechanical properties of Bamboo/PVC composite through hot-press molding. S.A Bahari [4] et al investigated the flexural strength, impact bending and water uptake of the composites by molding Bamboo/PVC composites from bamboo particle with various granular sizes and loadings.

The interface between bamboo powder and plastic is improved due to the molding pressure and holding time. the interface can improve the mechanical properties of BPC. Compared with injection molding and extrusion molding, BPC which made by compression molding has better mechanical properties. Compression molding holds advantages of high dimension precision, smooth surface, fine repetition et al, but it need more bigger mold if using compression molding to press great product, which increasing the cost of production, limiting the application of compression molding in BPC.

\section{Injection Molding}

Injection molding is a molding method by which the molten state of colloid inside nozzle through main runner into the mold cavity, then molding cooling as parts. As shown in Fig. 2, die assembly, and the nozzle is putted in the gate. After that, under the action of external force, molten state of BPC flow into the main runner through the nozzle, and then BPC flow the runner to the mold cavity. Finally, the mold of punch and die are opened, and the part is taken out. From Fig. 2, the cavity can be design as different shapes. It can ensure the high precision of thin-wall parts that the size of cavity is a constant when die assembly.

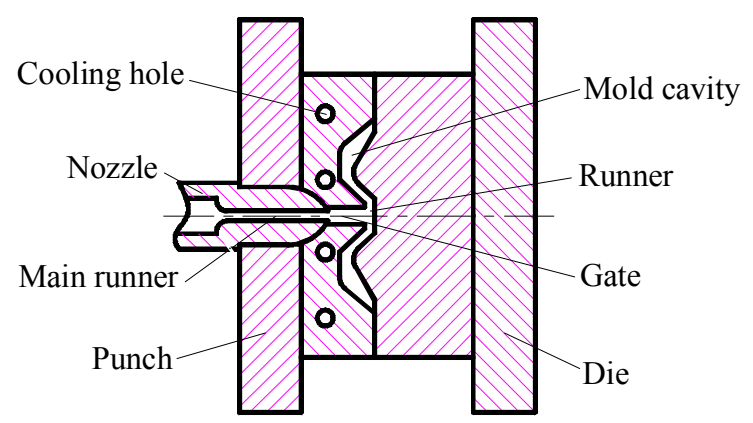

Fig. 2 Schematic drawing of injection molding.

The study of injection molding, mainly as: H. Liu [5] et al have demonstrated that bamboo powder can enhance the mechanical properties of bamboo/high-density polyethylene (HDPE) composite by HDPE, and composites had been injection molded into standard mechanical test specimens. S.K. Nayak [7] et al studied the effect of bamboo particle on the thermal stability and dynamic mechanical properties of BPC, which is obtained by injection molding. K Ohkita [7] et al researched the flexural properties of injection-molded bamboo/polybutylene succinate composite.

It is used more widely by the reason of injection molding holds advantages of a short molding cycle, high production efficiency and stable quality [8]. But, in the absence of external forces, the curing of BPC is poor, which results in the worse performance of BPC. In the process of injection, flow trace and pore is ease generated on the finished surfaces under the influence of BPC fluidity.

\section{Extrusion Molding}

Extrusion molding is a molding method by which under the action of the extrude barrel and screw, the plasticizing material molding as part through extrusion die. As illustrated in Fig. 3, in the cylinder, BPC molding as part through extrusion die under the plunger extrusion. C.W. Lou [9] et al 
studies has shown that the mechanical properties of BPC (extrusion grade) were better than that of the BPC (injection grade). A. Porras [10] et al have prepared BPC by extrusion molding. Extrusion molding can not guarantee the size of extrusion product as a constant by extrusion molding. And many factors affect extrusion molding, such as: nozzle temperature, the thermal flow of BPC and extrusion rate. So extrusion molding is less application in BPC.

But in recent years, Japanese scholars have studied the impact of extrusion molding on BPC. S. Kajikawa1[11] et al investigated the effect of the surface state on the fluidity of the bamboo powder. As illustrated in Fig. 4, bamboo powder is putted into the cylinder, and then the heater is opened. Finally, the sample was squeezed out by the piston through the die of capillary under the action of external load. It is a good research method to how to improve BPC liquidity and the mechanical properties of BPC by extrusion molding. All of them can provide the reference value for BPC in the application of 3D printing.

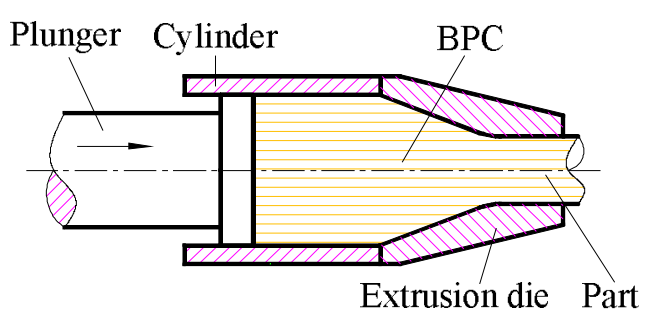

Fig. 3 Schematic drawing of Extrusion molding.

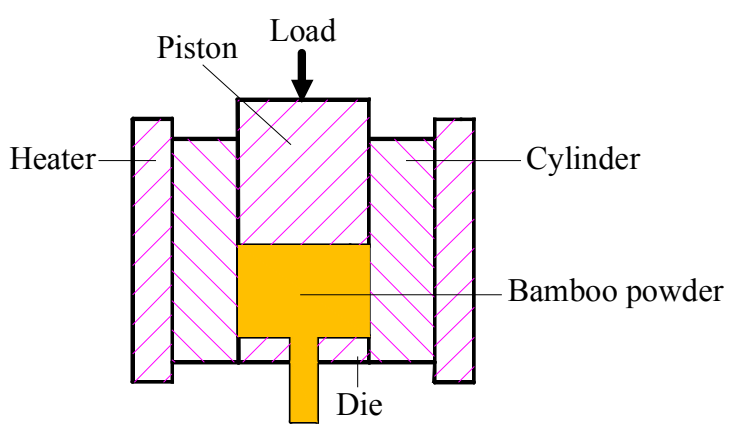

Fig.4 Schematic drawing of flow tester

\section{D Printing}

3D printing is a new technology, which is based on mathematical model file, and forming the metal powders or plastic as parts by overlapping layer by layer. Compared with other molding, 3D printing holds advantages of without die, has no relation to the space complexity, fast molding speed and no waste. D.J. Zhao [12] et al made bamboo powder/ polyamide (PA) blend by laser sintering. And the bending test results showed that bamboo powder/PA blend had good forming characteristic. Also the impeller had been shaped, as shown in Fig. 5, which illustrated that the material can be sintered complex parts. But it is influence the quality of parts that the bamboo powder/PA blend buckling deformation, which will be happen in the process of laser sintering.

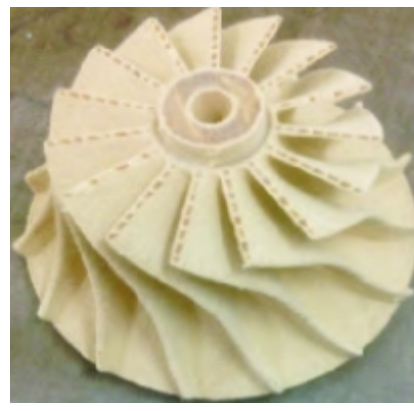

Fig.5 FMD Forming Impeller Part.

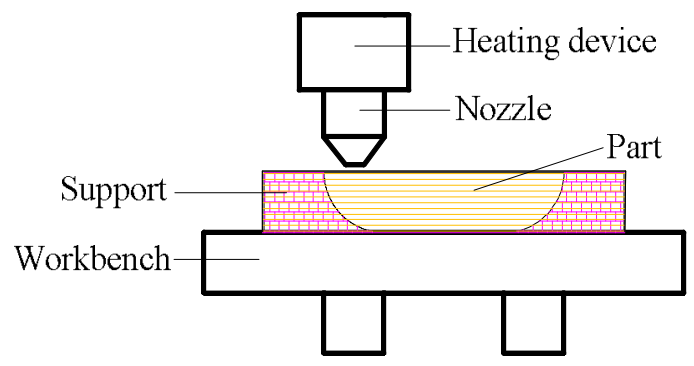

Fig.6 Device for FMD.

Fused deposition molding (FDM) is one of the 3D printing technologies. As shown in Fig. 6, material becomes into molten state under the action of heating device, and then the material flow from nozzle to support. Finally, part is molded on the workbench. FMD is very fit for BPC molding, and the material buckling deformation will not happen in the process of FMD. Although, there is rarely reported about FMD in the application of BPC, but wood/plastic composite has been molded 
by FMD. K. Luo [13] et al through improved the FDM equipment for wood/polyethylene molding, and mechanical properties of it is investigated.

\section{Conclusions}

To sum up, BPC molding will be in 3D printing technology development. Although, there is rarely reported about 3D printing in the application of BPC. But the application will be promoted that $\mathrm{BPC}$ is used to forming physics model by $3 \mathrm{D}$ printing. In the longer run, we believe that BPC will be wider application, which is a better solution to solve the problem of the lack of wood resources and the environment pollution of plastic.

\section{Acknowledgments}

The authors gratefully acknowledge the support of the Guangxi Natural Science Foundation (grant number 2013GXNSFAA019305) and Guangxi Key Laboratory of manufacturing System \& Advanced Manufacturing Technology (grant number 14-045-15-005Z).

\section{References}

[1] A.C. Manalo, et al, J. Composites Part B. 80 (2015) 73-83

[2] H. Ismail, et al, J. Polymer Testing. 21 (2002) 139-144

[3] H. Wang, et al, J. Journal of Bionic Engineering Suppl, (2008) 28-33

[4] Bahari. S.A, Krause. A, J. Journal of Cleaner Production, Xxx (2015) 1-9

[5] H. Liu , et al, J. Composites: Part A, 39 (2008) 1891-1900

[6] Sanjay K. Nayak, et al, J. Materials Science and Engineering A, 523 (2009) 32-38

[7] K. Ohkita, H. Takagi, J. International Journal of Modern Physics B, 24 (2010) 2838-2843

[8] Wang. R., et al, J. Part B: Physics, 52(2013b), 206-221

[9] C.W. Lou, et al, J. Journal of Materials Processing Technology, 192-193 (2007) 428-433

[10]A. Porras, A. Maranon, J. Composites: Part B, 43 (2015) 2782-2788

[11]S. Kajikawa, et al, J. Key Engineering Materials, 651-653 (2015) 830-835

[12]D.J Zhao, Y.L Guo, W.L Song, J. Journal Of Anhui Agri, 6 (2015) 184-186. (In Chinese)

[13]K. Luo, et al, J. Journal Of Northeast Forestry University, 38 (2010) 10. (In Chinese) 